\title{
Bases para la cuantificación de la huella de carbono de las repoblaciones forestales desde el enfoque del análisis de ciclo de vida
}

Basis for carbon footprint quantification of forestation processes from a life cycle analysis perspective

Alonso González, A. ${ }^{*}$; Oliet Palá, J.A. ${ }^{1}$; Rodríguez Olalla, A. ${ }^{2}$;

Álvarez Gallego, S. ${ }^{3}$; Rubio Sánchez, A. ${ }^{1}$

Departamento de Recursos y Sistemas Naturales.

Escuela Técnica Superior de Ingeniería de Montes, Forestal y del Medio Natural.

Universidad Politécnica de Madrid. C/ José Antonio Nováis 10. 28040 Madrid

${ }^{2}$ Departamento de Economía de la Empresa (ADO), Economía Aplicada II y Fundamentos de Análisis Económico. Facultad de Ciencias Jurídicas y Sociales.

Campus Madrid: Paseo de Artilleros s/n 28032. Madrid

${ }^{3}$ Departamento de Ingeniería y Morfología del Terreno.

Escuela Técnica Superior de Ingeniería de Caminos, Canales y Puertos.

C/ del Profesor Aranguren 3. 28040 Madrid,

*Autor para correspondencia: alex.alogon@gmail.com 


\section{Resumen}

Las repoblaciones forestales son una medida aceptada de compensación de las emisiones de gases de efecto invernadero (GEI) ampliamente utilizadas dentro de la acción climática. La capacidad secuestradora de $\mathrm{CO}_{2}$ de los bosques y su funcionalidad como grandes sumideros son las razones que hacen atractiva esta medida. No obstante, el proceso repoblador implica unas emisiones de GEI que por el momento no son tenidas en cuenta en los balances de carbono. Por ello, este trabajo estudia los procesos repobladores desde la perspectiva de la norma ISO 14067:2018 "Gases de efecto invernadero - Huella de carbono de productos - Requisitos y directrices para cuantificación" y facilitando lo necesario para su cumplimiento. Para ello, se han establecido los límites del sistema, inventariando todas las actividades emisoras de GEI; se han recopilado las fuentes de donde obtener los datos necesarios para aplicar el método de cálculo diseñado; y se han diseñado dos ejemplos de repoblación para utilizar la herramienta Excel creada, que cuantifica la huella de carbono (HC) de una repoblación según sus particulares características. Las emisiones de las repoblaciones utilizadas como casos de estudio han resultado en 559.38 y $654.71 \mathrm{~kg} \mathrm{CO}_{2}$ eq ha-1 , que corresponden respectivamente a una repoblación con una especie productora de papel, Eucalyptus globulus, y una repoblación multiusos mediterránea con dos especies de fagáceas y una de pino. Estos ejemplos tratan de cubrir la amplia variabilidad de las repoblaciones. Emisiones que suponen menos de un $1 \%$ de las absorciones potenciales totales que realizará la masa forestal creada. Por tanto, se establece que, desde una perspectiva climática, la rentabilidad de las repoblaciones es muy elevada y se confirma su conveniencia como medida de compensación frente al cambio climático e instrumento de primer orden como medida mitigadora del cambio climático.

Palabras clave: cambio climático, ciclo de vida, emisiones, mitigación, secuestro de carbono.

\section{Summary}

Reforestation is a recognized measure to offset greenhouse gas (GHG) emissions that is widely used in climate action. The $\mathrm{CO}_{2}$ sequestration capacity of forests and their function as large carbon sinks are the reasons why this measure is attractive. However, the reforestation process involves GHG emissions that are usually not yet considered in carbon balances. Therefore, this paper studies the forestation processes from the perspective of the norm ISO 14067:2018 "Greenhouse gases - Carbon footprint of products - Requirements and guidelines for quantification" and providing what is necessary for its compliance. In order to do so, the system limits have been established, listing all GHG emitting activities; the sources from which to obtain the necessary data to apply the designed calculation method have been collected; and two examples of reforestations have been designed to use the Excel tool created, which quantifies the carbon footprint of any forestation processes according to its specific characteristics. The emissions of the reforestations used as case studies have resulted in 559.38 and $654.71 \mathrm{~kg} \mathrm{CO}_{2}$ eq ha ${ }^{-1}$, that correspond respectively to a paper-producing reforestation with Eucalyptus globulus and a Mediterranean multipurpose reforestation with two Fagaceae species and a pine species. They try to cover the existing wide scope of possible reforestations. These emissions represent less than $1 \%$ of the total potential $\mathrm{CO}_{2}$ removals that will be made by the forest stand created. Therefore, it is established that, from a climate change perspective, the profitability of forestation processes is very high and its suitability as a compensation measure against climate change is reassured, as well as its preferential character.

Keywords: carbon sequestration, climate change, emissions, life cycle analysis, mitigation. 


\section{Introducción}

El cambio climático y el calentamiento global son los impactos ambientales más importantes a los que tiene que enfrentarse la humanidad hoy en día. Por ello la Organización de las Naciones Unidas ha definido una serie de Objetivos de Desarrollo Sostenible que determinan diferentes metas, entre las que aparecen con identidad propia las relacionadas directamente con el cambio climático. La Unión Europea materializa estos compromisos a través del Pacto Verde ("European Green Deal", 2020), donde se establecieron los objetivos a cumplir y las medidas y actuaciones que se consideran adecuadas para cumplirlos. Una de las metas es conseguir la neutralidad climática en la Unión Europea para 2050.

La neutralidad climática persigue lograr que las emisiones de gases de efecto invernadero (GEI) sean iguales a las remociones o absorciones de estos gases de la atmósfera. Para alcanzar la neutralidad climática, primero se deben mitigar las emisiones (reducirlas en origen) y, aquello que no se pueda mitigar ha de compensarse a posteriori. En este sentido, la $\mathrm{HC}$ es un indicador ambiental de sostenibilidad que mide el impacto potencial de las actividades humanas en cuanto a la generación de GEI con repercusión sobre el calentamiento global, que se expresa en masa equivalente de $\mathrm{CO}_{2}$.

La cuantificación de la HC se puede realizar conforme a diversas metodologías descritas en diferentes normas y estándares internacionales, como la ISO 14067:2018 "Gases de efecto invernadero. Huella de carbono de productos. Requisitos y directrices para cuantificación", que deriva de la metodología del análisis de ciclo de vida, establecida en las normas ISO 14040:2006 "Gestión ambiental. Análisis del ciclo de vida. Principios y marco de referencia” y la ISO 14044:2006 "Gestión ambiental. Análisis del ciclo de vida. Requisitos y directrices".

El Pacto Verde Europeo contempla los bosques como un importante sumidero de carbono dada su capacidad de fijación de carbono atmosférico, el cual acumulan en su biomasa y en el suelo (Liu et al., 2018). Consecuentemente, reconoce las actuaciones de forestación como medidas compensatorias de emisiones, dado que estas actuaciones, también llamadas repoblaciones forestales, incluyen tanto las forestaciones (creación de masas forestales en terreno previamente no forestal), como las reforestaciones (reintroducción de especies forestales en terrenos previamente forestales que quedaron rasos). A través de estas actuaciones se pretende mantener o aumentar la red de sumideros naturales de carbono, de la cual forman parte los bosques, océanos y el suelo (Falkowski, 2000).

Existen numerosos estudios sobre las absorciones de los bosques según las características de la vegetación (Taylor and Marconi, 2020), así como de los aprovechamientos (Lippke et al., 2011). No obstante, al estudio de las emisiones de GEI ligadas a los procesos repobladores todavía no se le ha prestado suficiente atención. Por ello, en el presente trabajo se han definido los siguientes objetivos: 
1. Aplicar la metodología de cuantificación de HC a las repoblaciones forestales.

2. Mejora del cálculo de los balances de carbono de las actuaciones de forestación.

3. Definir el ciclo de vida de la repoblación forestal estableciendo los límites del sistema e identificando las actividades emisoras de gases de efecto invernadero.

4. Recopilar fuentes que aportan datos sobre sus emisiones.

5. Elaborar una herramienta de cálculo aplicable a los procesos repobladores de características seleccionables.

\section{Materiales y métodos}

\subsection{Perspectiva de Análisis de Ciclo de Vida}

De acuerdo con la norma ISO 14067:2018, la metodología del análisis del ciclo de vida se estructura en cuatro fases:

1. Definición del objetivo y alcance.

2. Análisis de inventario de ciclo de vida.

3. Evaluación del impacto del ciclo de vida.

4. Interpretación del ciclo de vida.

Esta metodología se rige por una serie de principios entre los que destacan:

- Enfoque iterativo, por el cual cada fase se reevalúa continuamente al ser interdependientes.

- Enfoque relativo, la cuantificación se hace en referencia a la unidad funcional (medida básica del sistema estudiado que permite compararlo con otros sistemas).

- Evasión de la doble contabilidad de las emisiones, asignándolas de forma única y específica a una fase.

El enfoque de análisis de ciclo de vida para un producto tiene en cuenta las siguientes cinco etapas:

1. Adquisición de materia prima.

2. Producción. 
3. Distribución y transporte.

4. Uso y mantenimiento.

5. Tratamiento de residuos al final de la vida útil.

\subsection{Definición del alcance del sistema}

En el caso que nos ocupa, la unidad funcional que ha sido considerada es una hectárea de rodal de repoblación, con una serie de características variables y seleccionables por el repoblador, mientras que los límites del sistema (véase Figura 1), definidos por los límites de cada etapa del ciclo de vida, son:

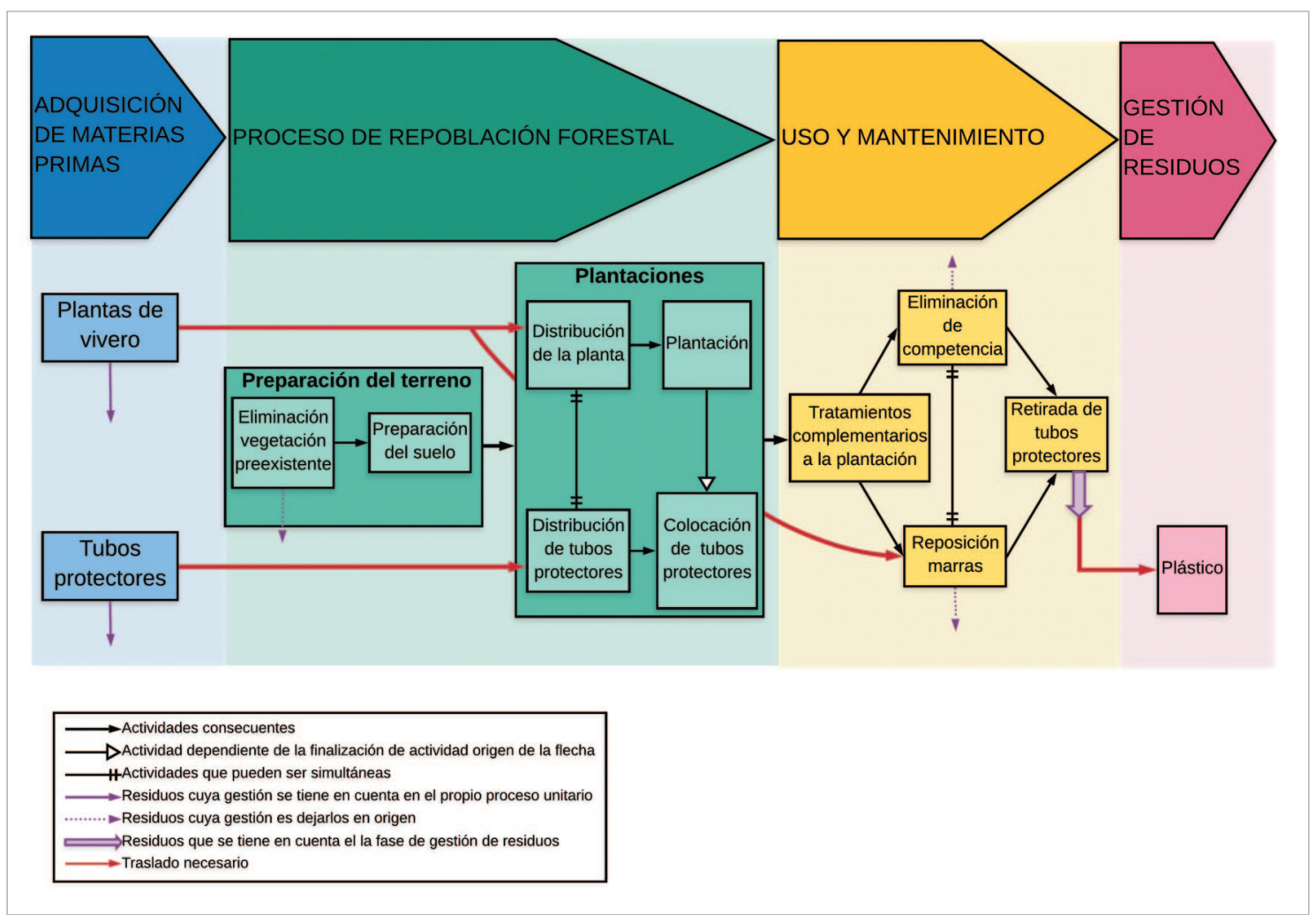

Figura 1. Mapa de procesos incluidos en el sistema estudiado de la repoblación forestal para cuantificar la huella de carbono.

- Adquisición de materia prima: incluye plantas de vivero y los tubos protectores (considerados por su capacidad de aumentar la supervivencia de las plantas y de disminuir el consumo de agua de riego en las repoblaciones (Arnold and Alston, 2012)). Las actividades incluidas son la obtención de recursos para la producción y empaquetado de la materia prima, el transporte de los recursos al lugar de producción y empaquetado, la producción y empaquetado de la materia prima para su transporte, además de la gestión y tratamiento de los residuos producidos. 
- Producción (repoblación forestal): tiene en cuenta el traslado de la materia prima, de personal y maquinaria al lugar que se va a repoblar, la preparación del terreno (eliminación de vegetación preexistente y el tratamiento del suelo) y la propia plantación (distribución e instalación de la planta y del tubo protector).

- Etapa de uso y mantenimiento: tiene en cuenta el mismo tipo de traslados que la etapa de repoblación, pero las actividades incluidas son los cuidados culturales de la plantación (alcorques, castilletes, aporte de abono, desbroces selectivos, reposición de marras y retirada de tubos protectores).

- Gestión de residuos: incluye el traslado de los tubos protectores retirados a la central de tratamiento de residuos y el propio tratamiento en planta de residuos.

\subsection{Procedencia y calidad de datos}

Los inventarios de Ecoinvent (Wernet et al., 2016) se utilizan como modelo para el cálculo de los factores de emisiones de distintos procesos y para la categorización de materiales según la clasificación de Ecoinvent, dada la alta calidad de sus datos, aunque la cobertura temporal, geográfica o tecnológica no sea siempre la más adecuada. Los factores de emisión se obtienen a través del programa SimaPro, donde se han considerado las siguientes condiciones:

- Se desprecian las emisiones de las actividades que representan menos de un $0.1 \%$ del total de emisiones.

- Se excluyen las emisiones a largo plazo y las originadas por las infraestructuras.

Por otro lado, la aportación más novedosa de este trabajo es la utilización de las tarifas de TRAGSA (Grupo TRAGSA, 2020) para poder calcular las emisiones de GEI de las actividades forestales. Las tarifas consisten en una recopilación de unidades de obra que se pueden contratar a la hora de realizar una repoblación forestal, y contemplan el rendimiento de la maquinaria y el personal, es decir, el tiempo que se requiere para realizar esa unidad de obra, cuya unidad referencial puede ser puntual, lineal o de superficie. A partir de estos rendimientos se han diseñado las ecuaciones del método de cálculo recogido en el epígrafe 3.1. y se han buscado los datos necesarios para relacionarlos con la unidad funcional del sistema, como el consumo horario de la maquinaria.

Por otra parte, el uso de un programa de optimización de cargas, en concreto Easycargo, es necesario para calcular la cantidad de vehículos requeridos para transportar una cantidad específica de materiales de ciertas medidas, y poder aplicar así los factores de emisión adecuados al tamaño de los vehículos.

Se recogen las fuentes más importantes en la Tabla 1 . 
Tabla 1. Fuentes de datos utilizados en el trabajo y análisis de calidad.

\begin{tabular}{|c|c|c|}
\hline Fuente & Descripción de fuente & Datos obtenidos \\
\hline Ecoinvent & $\begin{array}{l}\text { Base de datos suiza que proporciona análisis } \\
\text { de ciclo de vida consistentes y transparentes, } \\
\text { bien documentados, para miles de productos. }\end{array}$ & $\begin{array}{l}\text { Emisiones de procesos } \\
\text { de fabricación y factores } \\
\text { de emisión de traslados }\end{array}$ \\
\hline MITECO & $\begin{array}{l}\text { Emisores de documentación para regular } \\
\text { el cómputo de emisiones y HC. }\end{array}$ & $\begin{array}{l}\text { Factores de emisiones } \\
\text { de combustibles, gestión } \\
\text { de residuos y de mix } \\
\text { de electricidad }\end{array}$ \\
\hline $\begin{array}{l}\text { Tarifas TRAGSA } \\
\text { (público) }\end{array}$ & \multirow{2}{*}{$\begin{array}{l}\text { Empresa pública técnica } \\
\text { de referencia en el sector forestal } \\
\text { de España. }\end{array}$} & $\begin{array}{l}\text { Rendimientos de maquinaria } \\
\text { y personal para actividades de } \\
\text { repoblación y uso y mantenimiento }\end{array}$ \\
\hline $\begin{array}{l}\text { TRAGSA } \\
\text { (comunicación } \\
\text { personal) }\end{array}$ & & $\begin{array}{c}\text { Consumos horarios de maquinaria } \\
\text { utilizada en actividades }\end{array}$ \\
\hline
\end{tabular}

\section{Resultados}

\subsection{Método de cálculo}

A partir de los datos recopilados y las consideraciones realizadas se ha diseñado el método de cálculo que permite cuantificar las emisiones de GEI generadas en cualquier repoblación forestal. La Tabla 2 recoge un resumen de todas las actividades que producen GEI y, por tanto, de las ecuaciones que comprenden el método de cálculo diseñado. Asimismo, las ecuaciones desarrolladas están representadas en las Figuras 2-10, que se han diseñado en torno a las unidades de referencia de los factores de emisiones.

Es importante destacar que las ecuaciones diseñadas para el proceso de repoblación son también aplicables a la etapa de uso y mantenimiento, dado que las emisiones proceden del mismo tipo de actividades. Asimismo, se han establecido ciertas consideraciones que se ven reflejadas en las fórmulas:

— Número de trayectos del personal serán dos por día (ida y vuelta).

- Número de trayectos de la maquinaria serán dos, uno al inicio de las actividades y otro al final.

- Los residuos de tubos protectores suponen un $60 \%$ en masa del total de los instalados (Arnold and Alston, 2012).

Por último, las emisiones tendrán distintos orígenes según la actividad, como se puede ver en la Figura 12. En las flechas rojas, actividades cuyas emisiones GEI se contabilizan en la actividad destino. Estas emisiones incluyen las emitidas por el 
Tabla 2. Simbología de las emisiones de cada etapa utilizada en las figuras.

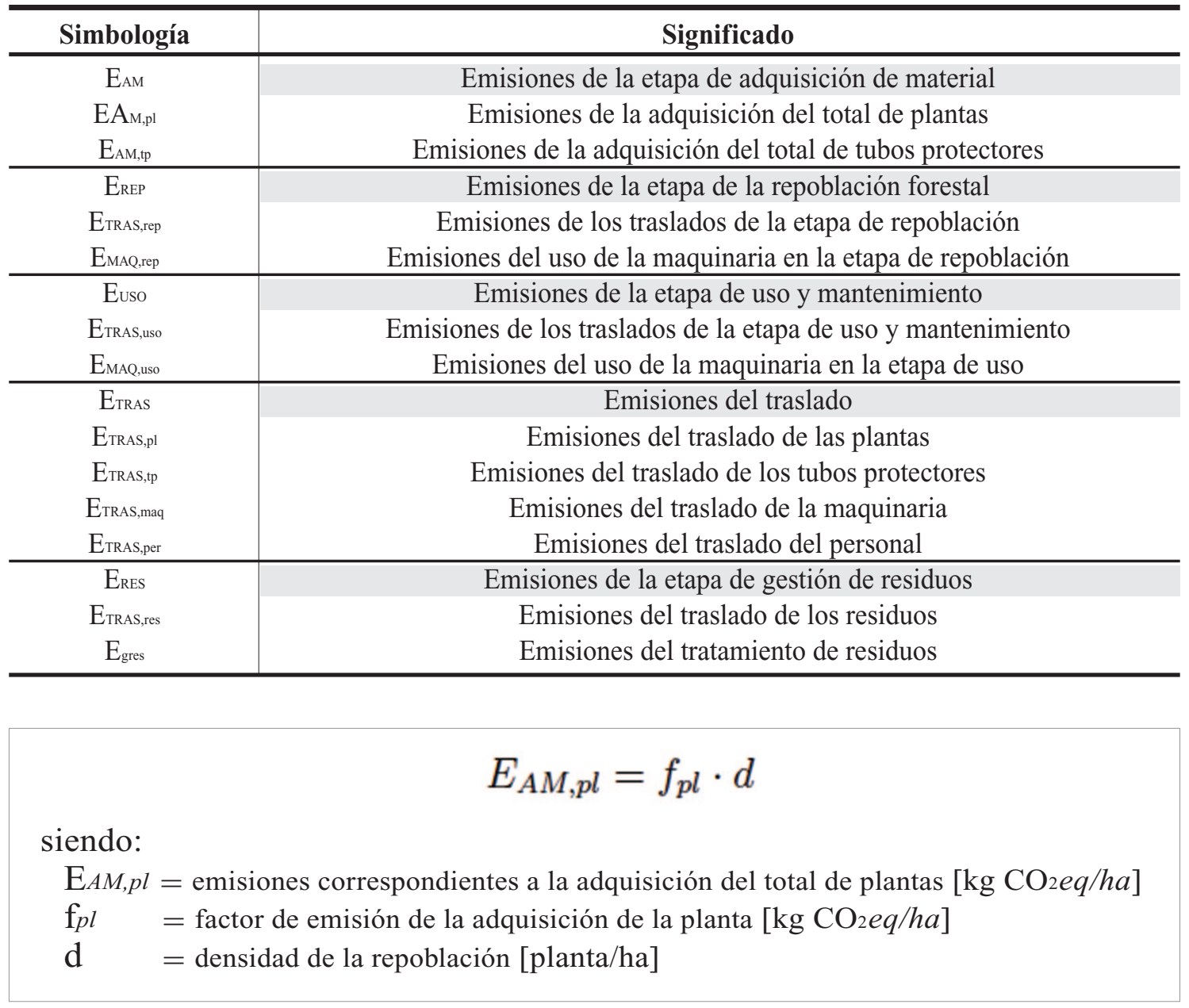

Figura 2. Ecuación para calcular las emisiones correspondientes a la adquisición del total de plantas.

$$
E_{A M, t p}=f_{t p} \cdot \frac{P_{t p}}{10^{6}} \cdot d \cdot \alpha
$$

siendo:

$\mathrm{E}_{A M, p l}=$ emisiones correspondientes a la adquisición del total de tubos protector $[\mathrm{kg} \mathrm{CO}$ eq/ha]

$\mathrm{f}_{t p}=$ factor de emisión de la adquisición del tubo protector [ $\mathrm{kg} \mathrm{CO}$ eq/ton de tubos]

$P_{t p} \quad=$ peso del tubo protector [g/tubo]

$\mathrm{d}=$ densidad de la repoblación [planta/ha]

$\alpha=$ proporción de plantas entubadas [\%]

Figura 3. Ecuación para calcular las emisiones correspondientes a la adquisición del total de tubos protectores. 


$$
\begin{gathered}
E_{T R A S, p l}=\sum_{i} \frac{f_{V, i} \cdot D_{p l} \cdot P_{b d} \cdot\left[\left(N_{V, i}-1\right) \cdot N_{T, b d, V, i}+N_{t, b d, V, i}\right]}{S} \\
N_{T, b d}=\frac{d \cdot S}{N_{a l v}}=\sum_{i} N_{T, b d, i}
\end{gathered}
$$

siendo:

$\mathrm{E} T R A S, p l=$ emisiones correspondientes al traslado de plantas $[\mathrm{kg} \mathrm{CO} 2 e q / h a]$

$\mathrm{f}_{V, i}=$ factor de emisión de transporte de vehículo de tipo i $[\mathrm{kg} \mathrm{CO}$ eq/ $/ \mathrm{km}]$

$\mathrm{D}_{p l}=$ distancia entre el vivero y la repoblación $[\mathrm{km}]$

$\mathrm{P} b d \quad=$ peso de una bandeja forestal [ton]

$\mathrm{N}_{V, i} \quad=$ número de vehículos de tipo i [ud]

$\mathrm{N} T, b d, V, i=$ número total de bandejas forestales en vehículo de tipo i lleno [ud]

$\mathrm{N}_{t, b d, V, i}=$ número total de bandejas forestales en vehículo de tipo i vacío [ud]

$\mathrm{S} \quad=$ superficie de la repoblación [ha]

$\mathrm{N} T, b d=$ número total de bandejas forestales [ud]

$\mathrm{N}$ alv = número de alveolos de las bandejas forestales [ud]

$\mathrm{d}=$ densidad de la repoblación [planta/ha]

$\mathrm{N}_{T, b d, i}=$ número total de bandejas forestales en vehículo i [ud]

Figura 4. Ecuaciones para calcular las emisiones correspondientes al traslado de plantas.

$$
\begin{gathered}
E_{T R A S, t p}=\sum_{i} \frac{f_{V, i} \cdot D_{t p} \cdot P_{t p} \cdot\left[\left(N_{V, i}-1\right) \cdot N_{T, t p, V, i}+N_{t, t p, V, i}\right]}{S} \\
N_{T, t p}=d \cdot S \cdot \alpha=\sum_{i} N_{T, t p, i}
\end{gathered}
$$

siendo:

ETRAS,tp $=$ emisiones correspondientes al traslado de tubos $[\mathrm{kg} \mathrm{CO} e q / h a]$

$\mathrm{f}_{V, i} \quad=$ factor de emisión de transporte de vehículo de tipo i $[\mathrm{kg} \mathrm{CO} 2 \mathrm{eq} / \mathrm{tkm}]$

$\mathrm{D}_{t p} \quad=$ distancia entre la fábrica de tubos protectores y la repoblación $[\mathrm{km}]$

$\mathrm{P}_{t p} \quad=$ peso de un tubo protectos [ton]

$\mathrm{N} V, i=$ número de vehículos de tipo i [ud]

$\mathrm{N} T, t p, V, i=$ número total de tubos protectores en vehículo de tipo i lleno [ud]

$\mathrm{N}_{t, t p, V, i}=$ número total de tubos protectores en vehículo de tipo i vacío [ud]

$\mathrm{S} \quad=$ superficie de la repoblación [ha]

$\mathrm{N} T, t p, i=$ número total de tubos protectores en vehículo i [ud]

$\mathrm{N} T, t p \quad=$ número total de tubos protectores [ud]

$\mathrm{d}=$ densidad de la repoblación [planta/ha]

$\alpha \quad=$ proporción de plantas entubadas [\%]

Figura 5. Ecuaciones para calcular las emisiones correspondientes al traslado de tubos protectores. 


$$
\begin{gathered}
E_{T R A S, p e r}=\frac{f_{c a r} \cdot D_{c a r} \cdot \sum\left(N_{c a r, a c t, i} \cdot N_{\text {tray }, c a r, a c t, i}\right)}{S} \\
N_{\text {tray }, c a r}=2 \cdot t_{f i n} \\
N_{c a r}=\frac{\frac{N_{p e r}}{7}+N_{p e r}}{C a r} \\
N_{\text {per }}=\frac{N_{\text {act }, i} \cdot \eta_{\text {act }, i}}{t_{\text {fin }} \cdot t_{\text {jor }}}
\end{gathered}
$$

siendo:

ETRAS,per = emisiones correspondientes al traslado de personal $\left[\mathrm{kg} \mathrm{CO}_{2} e q / h a\right]$

$\mathrm{f}_{\text {car }} \quad=$ factor de emisión del coche $[\mathrm{kg} \mathrm{CO} 2 \mathrm{eq} / \mathrm{km}]$

$\mathrm{N}_{\text {car }, \text { act, } i}=$ número de coches para la actividad i [ud]

Dcar = distancia entre la oficina y la repoblación $[\mathrm{km}]$

$\mathrm{N}_{\text {tray }}$ car, act, $i=$ número de trayectos de coche con personal para la actividad i [ud]

$\mathrm{S} \quad \quad=$ superficie de la repoblación [ha]

t fin $\quad=$ tiempo deseado para el término de las tareas [días]

Nper $\quad=$ número total de peones [ud]

Car $\quad=$ número de pasajeros en un coche [ud]

Nact, $i=$ número total de unidades de obra de la actividad i [ud]

$\eta$ act, $i=$ rendimiento de la actividad $\mathrm{i}[\mathrm{h} / \mathrm{ud}]$

tior $\quad=$ duración de la jornada de trabajo [h/día]

Figura 6. Ecuaciones para calcular las emisiones correspondientes al traslado del personal 


$$
\begin{gathered}
E_{M A Q}=\frac{E_{M A Q, p r o d}+E_{M A Q, a c t}+E_{M A Q, f i n}}{S} \\
E_{M A Q, p r o d}=\sum_{i} \frac{f_{\text {prod }, i} \cdot N_{a c t, i} \cdot \eta_{a c t, i}}{v i d a_{u} t i l, i} \\
E_{M A Q, f i n}=\sum_{i} \frac{f_{\text {fin }, i} \cdot N_{\text {act }, i} \cdot \eta_{a c t, i}}{v i d a_{\text {util }, i}} \\
E_{M A Q, a c t}=\sum_{i} f_{c} \cdot c_{h, i} \cdot N_{a c t, i} \cdot \eta_{a c t, i}
\end{gathered}
$$

siendo:

$\mathrm{E} M A Q=$ emisiones correspondientes al uso de la maquinaria $[\mathrm{kg} \mathrm{CO} e q / h a]$

$\mathrm{E} M A Q$, peod $=$ emisiones alícuotas de la producción de la maquinaria $[\mathrm{kg} \mathrm{CO} e q]$

$\mathrm{E} M A Q, a c t=$ emisiones correspondientes la actividad de la maquinaria $\left[\mathrm{kg} \mathrm{CO}_{2} e q\right]$

$\mathrm{E} M A Q$, fin $=$ emisiones alícuotas del término de la maquinaria $[\mathrm{kg} \mathrm{CO} 2 e q]$

$\mathrm{S} \quad=$ superficie de la repoblación [ha]

$\mathrm{f}_{\text {prod, } i}=$ factor de emisión de la producción de la maquinaria de tipo i $[\mathrm{kg} \mathrm{CO}$ eq/ud]

$\mathrm{N}_{a c t, i}=$ número total de unidades de obra de la actividad i [ud]

$\eta$ act, $i=$ rendimiento de la actividad $\mathrm{i}[\mathrm{h} / \mathrm{ud}]$

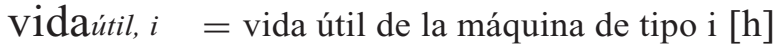

$\mathrm{f}_{\text {fin }, i}=$ factor de emisión del término de la máquina de tipo i $\left[\mathrm{kg} \mathrm{CO}_{2} e q / u d\right]$

$\mathrm{f}_{c} \quad=$ factor de emisiones del gasóleo $\mathrm{B}[\mathrm{kg} \mathrm{CO}$ eq/l]

$\mathrm{c} h, i=$ consumo horario de la máquina de tipo i $[1 / \mathrm{h}]$

Figura 7. Ecuaciones para calcular las emisiones correspondientes al uso de la maquinaria. 


$$
\begin{gathered}
E_{T R A S, \text { maq }}=N_{\text {tray }, \text { maq }} \cdot \sum_{i} \frac{f_{\text {gon }} \cdot N_{\text {maq }, i} \cdot D_{\text {maq }} \cdot P_{\text {maq }, i}}{S} \\
N_{\text {maq }, i}=\frac{N_{a c t, i} \cdot \eta_{\text {act }, i}}{t_{\text {fin }} \cdot t_{\text {jor }}}
\end{gathered}
$$

siendo:

ETRAS,maq = emisiones correspondientes al transporte de la maquinaria $\left[\mathrm{kg} \mathrm{CO} \mathrm{CO}_{2}\right.$ /ha $]$

$\mathrm{N}_{\text {tray, } m a q} \quad$ = número de trayectos para traslado de maquinaria [ud]

$\mathrm{fgon}_{\text {gon }}=$ factor de emisión de transporte de máquina con góndola $\left[\mathrm{kg} \mathrm{CO}_{2} e q / \mathrm{tkm}\right]$

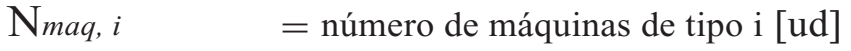

D maq $=$ distancia entre el almacén de máquinas y la repoblación $[\mathrm{km}]$

P maq, $i=$ peso de la máquina de tipo i [ton]

$\mathrm{S} \quad=$ superficie de la repoblación [ha]

$\mathrm{N}_{\text {maq }, i} \quad=$ número total máquina de tipo i [ud]

N tact, $i=$ número total de unidads de obra de la actividad $\mathrm{i}$ [ud]

$\eta$ act, $i=$ rendimiento de la actividad $\mathrm{i}[\mathrm{h} / \mathrm{ud}]$

$\mathrm{t}$ fin $\quad=$ tiempo deseado para finalización de las tareas [días]

tjor $\quad=$ duración de la jornada de trabajo [h/día]

Figura 8. Ecuaciones para calcular las emisiones correspondientes al traslado de la maquinaria.

$$
\begin{gathered}
E_{T R A S, \text { res }}=\frac{f_{\text {car }} \cdot N_{\text {car }, \text { res }} \cdot D_{\text {res }}}{S} \\
N_{\text {car,res }}=\frac{0.6 \cdot N_{T, t p}}{\text { cupo }_{c a r}}
\end{gathered}
$$

siendo:

ETRAS,res = emisiones correspondientes al traslados de residuos de tubos $[\mathrm{kg} \mathrm{CO}$ eq/ha $]$

$\mathrm{f}_{\text {car }} \quad=$ factor de emisión del coche $[\mathrm{kg} \mathrm{CO} 2 \mathrm{eq} / \mathrm{km}]$

$\mathrm{N}_{\text {car }}$ res = número de coches para el traslado de residuos [ud]

Dres = distancia entre la repoblación y el centro de tratamiento de residuos [km]

$\mathrm{S} \quad=$ superficie de la repoblación [ha]

$\mathrm{N} T, t p \quad=$ número total de tubos protectores [ud]

cupocar $\quad=$ cupo de tubos protectores que caben en un coche [ud]

Figura 9. Ecuaciones para calcular las emisiones correspondientes al traslado de residuos de los tubos protectores. 


$$
E_{\text {gres }}=\frac{0.6 \cdot f_{\text {gres }} \cdot P_{t p} \cdot N_{T, t p}}{S}
$$

siendo:

Egres = emisiones correspondientes a la gestión de residuos de tubos $\left[\mathrm{kg} \mathrm{CO}_{2} e q / h a\right]$

fgres, $i=$ factor de emisión del tratamiento de residuos plásticos [kg $\mathrm{CO}_{2}$ eq/ton]

$\mathrm{P}_{t p} \quad=$ peso total de un tubo protector [ton]

$\mathrm{N} T, t p \quad=$ número total de tubos protectores [ud]

$\mathrm{S} \quad=$ superficie de la repoblación [ha]

Figura 10. Ecuación para calcular las emisiones correspondientes a la gestión de residuos de tubos.

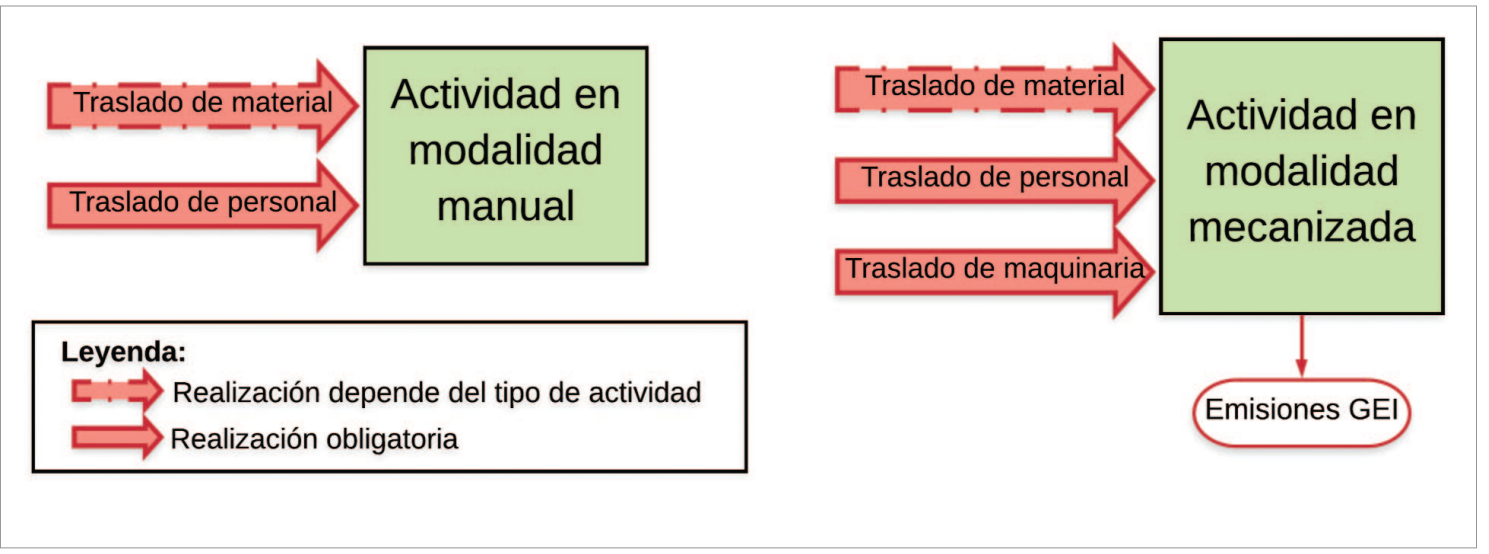

Figura 12. Origen de las emisiones GEI según la modalidad de las actividades de la repoblación forestal. En las flechas rojas, actividades cuyas emisiones GEI se contabilizan en la actividad destino. Estas emisiones incluyen las emitidas por el consumo de combustible de los vehículos, y la parte alícuota de las emisiones por producción y término de vida del vehículo.

consumo de combustible de los vehículos, y la parte alícuota de las emisiones por producción y término de vida del vehículo.

\subsection{Cálculo de la HC y absorciones. Ejemplos}

Con el método de cálculo descrito se ha elaborado una hoja de cálculo que está estructurada en tres hojas principales: entradas, actividades y resultados. La hoja "Entradas" incluye las tablas y menús desplegables para introducir los datos de las características de la repoblación y de los materiales utilizados, así como los datos a introducir en el programa Easycargo y sus correspondientes resultados. A través de lo introducido en esa primera hoja, aparecerá en "Actividades" la descripción de la unidad de obra correspondiente en las tarifas TRAGSA, donde habrá que consultar el rendimiento para introducirlo en la segunda hoja.

Se diseñaron dos ejemplos de repoblaciones forestales para cuantificar la $\mathrm{HC} \mathrm{a}$ 


\section{Reparto de emisiones por etapas del ciclo de vida y procesos unitarios de la repoblación productora de papel de Eucalyptus globulus.}

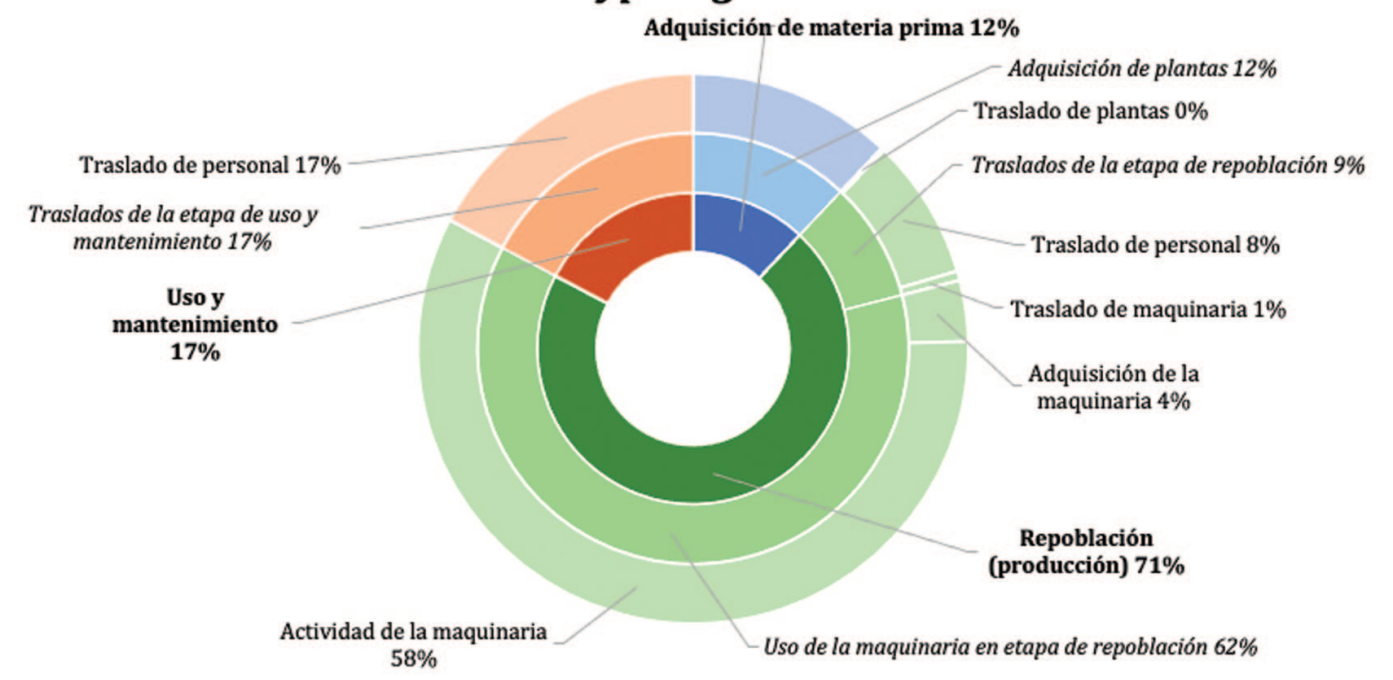

Figura 13. Representación del reparto de emisiones por etapas del ciclo de vida y procesos unitarios de la repoblación productora de eucalipto diseñada en el trabajo.

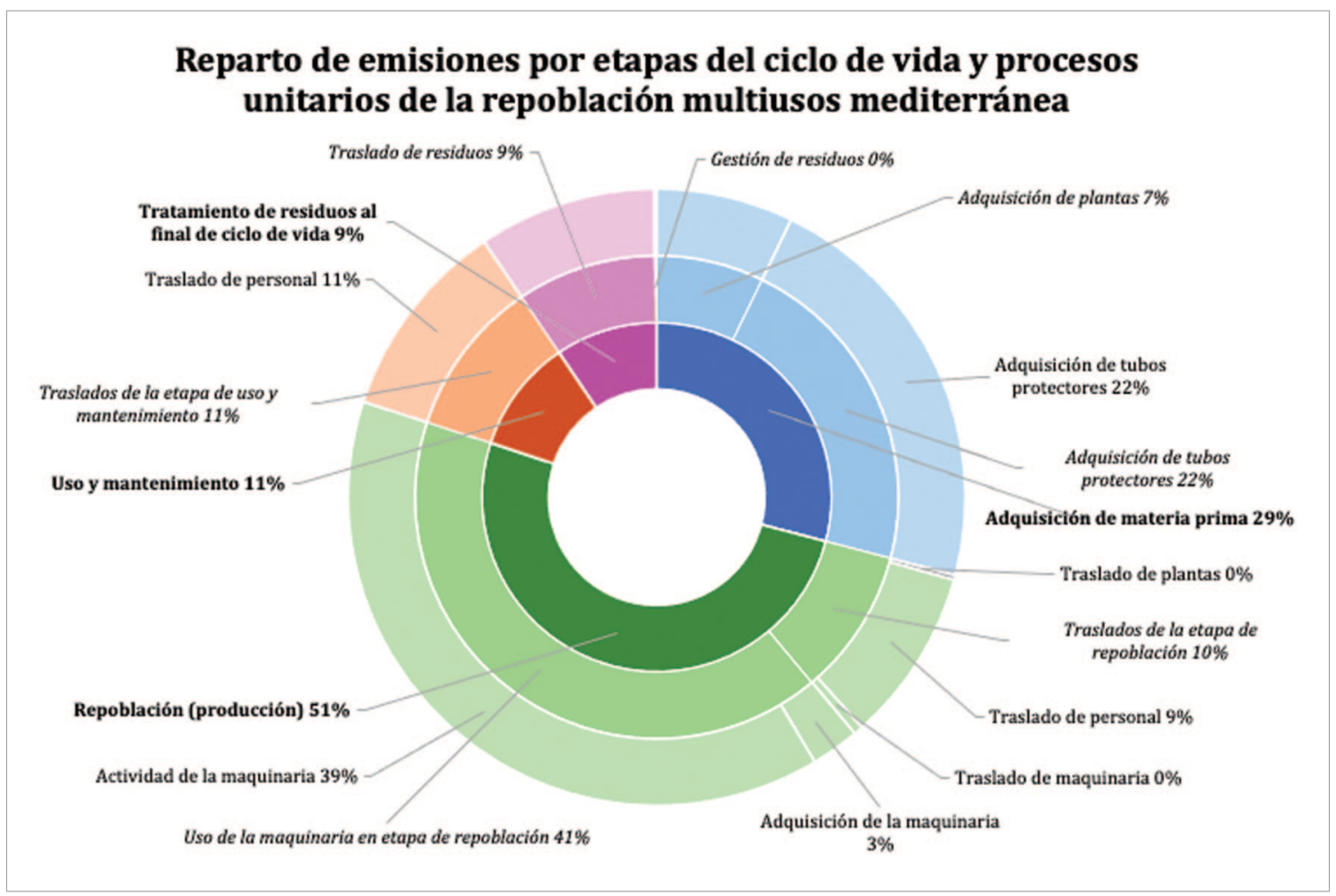

Figura 14. Representación del reparto de emisiones por etapas del ciclo de vida y procesos unitarios de la repoblación multiusos mediterránea diseñada en el trabajo. 
través de esta herramienta desarrollada. Por un lado, se ha considerado una repobla-

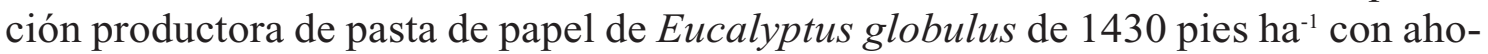
yado mecanizado con subsolador y eliminación química de la vegetación competidora de forma anual durante los dos primeros años (Serrada et al., 2008), resultando una $\mathrm{HC} 559.38 \mathrm{~kg} \mathrm{CO}_{2}$ eq ha ${ }^{-1}$. Por otro lado, se ha considerado una repoblación de uso múltiple en área mediterránea compuesta por un $60 \%$ de especies fagáceas y un $40 \%$ de pinos con una densidad de 1000 pies ha $^{-1}$. Esta repoblación requiere un acaballonado superficial, la implantación de tubos protectores a las fagáceas, la retirada de dichos tubos y un desbroce manual con desbrozadora el segundo año, y su HC supone $654.71 \mathrm{~kg} \mathrm{CO}_{2}$ eq ha' ${ }^{-1}$.

Los resultados obtenidos (véase Tabla 3. Rentabilidad de la HC de las repoblaciones ejemplo según las tasas de captura de las repoblaciones de Registro de Huella de Carbono. Años de permanencia: de las masas: 40. (Figura 13). Representación del reparto de emisiones por etapas del ciclo de vida y procesos unitarios de la repoblación productora de eucalipto diseñada en el trabajo. (Figura 14). Representación del reparto de emisiones por etapas del ciclo de vida y procesos unitarios de la repoblación multiusos mediterránea diseñada en el trabajo.) reflejan que la etapa con mayor aportación de emisiones a la $\mathrm{HC}$ es la repoblación (producción), superando el 50\% en ambos casos estudiados. Dentro de esta etapa, destaca la actividad de la maquinaria, que supone más del $80 \%$ de sus emisiones. Por otro lado, las emisiones de la adquisición de materiales aumentan en gran medida al utilizar tubos protectores, pasando del 12\% de la repoblación productora sin tubos al $29 \%$ de la repoblación mediterránea con tubos.

Tabla 3. Rentabilidad de la HC de las repoblaciones ejemplo según las tasas de captura de las repoblaciones de Registro de Huella de Carbono. Años de permanencia: de las masas: 40

\begin{tabular}{|c|c|c|}
\hline Repoblación ejemplo & Productora* & Multiusos \\
\hline $\mathrm{HC}\left(\mathrm{kg} \mathrm{CO} \mathrm{eq} \mathrm{ha}^{-1}\right)$ & 559.38 & 654.71 \\
\hline Absorciones anuales ( $\mathrm{t} \mathrm{CO}_{2}$ ha- $\left.1 \mathrm{año}^{-1}\right)$ & 10.18 & 2.75 \\
\hline Proporción HC frente a absorciones anuales ( $\%)$ & 5.50 & 23.81 \\
\hline Proporción HC frente a absorciones totales ( $\%)$ & 0.14 & 0.59 \\
\hline Tiempo de amortiguación de $\mathrm{HC}$ & 20 días & 2 meses y 26 días \\
\hline Amortiguación del impacto al final del periodo (pies ha-1) & 2 & 6 \\
\hline
\end{tabular}

*Turno de corta a hecho de 20 años.

Además, la Tabla 5. Rentabilidad de la HC de las repoblaciones ejemplo según las tasas de captura de las repoblaciones de Registro de Huella de Carbono. Años de permanencia: de las masas: 40. Recoge las absorciones calculadas con la herramienta del Registro de Huella de Carbono del Ministerio de Transición Ecológica, que son desde 4 a 20 veces mayores que la $\mathrm{HC}$ de la repoblación mediterránea y productora, respectivamente, tan solo en un año. 


\section{Discusión}

Como se ha mencionado anteriormente, la actividad de la maquinaria produce la mayor aportación de emisiones. De forma evidente, se debe al gran consumo de combustible y el tiempo que están funcionando. En caso de querer seleccionar la actividad mecanizada de menor impacto, deberán compararse los rendimientos, que deben estar expresados idealmente en términos de rendimientos puntuales. Esta transformación requiere que se comparen para una misma densidad de plantación. En la Tabla 4 se recoge el análisis de sensibilidad de las actividades estudiadas, del cual se extrae que, a menores densidades, las actividades puntuales son preferibles, y viceversa.

Tabla 4. Comprobación de la variable que causa la variación de la HC dentro de las emisiones por la actividad de maquinaria.

\begin{tabular}{|c|c|c|c|c|c|c|}
\hline \multirow{2}{*}{ 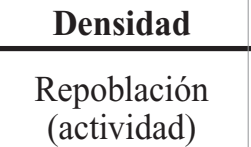 } & \multicolumn{3}{|c|}{1000 pies/ha } & \multicolumn{3}{|c|}{$1000 \mathrm{pies} / \mathrm{ha}$} \\
\hline & $\begin{array}{l}\text { Producción } \\
\text { (subsolado) }\end{array}$ & & $\begin{array}{c}\text { Multiusos } \\
\text { (acaballonado lineal) }\end{array}$ & $\begin{array}{l}\text { Productora } \\
\text { (subsolado) }\end{array}$ & & $\begin{array}{c}\text { Multiusos } \\
\text { (acaballonado lineal) }\end{array}$ \\
\hline $\begin{array}{c}\text { Emisiones } \\
\text { de la actividad } \\
\text { de la maquinaria } \\
\left(\mathrm{kg} \mathrm{CO}_{2} / \mathrm{ha}\right)\end{array}$ & 227.00 & $<$ & 252.48 & 227.00 & $>$ & 301.93 \\
\hline $\begin{array}{l}\text { Rendimiento } \\
(\mathrm{h} / \mathrm{mil})\end{array}$ & 4.8 & $<$ & $5.6^{*}$ & 4.8 & $>$ & $4.75^{*}$ \\
\hline $\begin{array}{l}\text { Consumo } \\
\text { de la máquina } \\
(1 / \mathrm{h})\end{array}$ & 17.5 & $>$ & 16.38 & 17.5 & $>$ & 16.38 \\
\hline
\end{tabular}

*transformado de lineal a puntual

Fuente: tarifas TRAGSA.

En lo que respecta a las emisiones de la adquisición de materiales, el componente principal de los tubos protectores es el polipropileno, un tipo de plástico, cuya adquisición supone el $85 \%$ de las emisiones producidas en el sistema de fabricación de los tubos (véase Tabla 5). Se recomienda reducir su aportación a la HC sustituyendo el material principal por otro que tenga menor impacto sobre el cambio climático. No obstante, el uso de los tubos supone ventajas para el éxito de la repoblación, mencionados en el epígrafe 2.2. 
Tabla 5. Recolección de los datos necesarios para calcular las emisiones totales del proceso unitario de adquisición de una tonelada de tubos protectores.

\begin{tabular}{c|c|c|c|c}
\hline Inventario & Ud & Cantidad & $\begin{array}{c}\text { Emisiones unitarias } \\
\left(\mathrm{kg} \mathrm{CO}_{2} \text { eq } / \mathrm{ud}\right)\end{array}$ & $\begin{array}{c}\text { Emisiones totales } \\
\left(\mathrm{kg} \mathrm{CO} \mathrm{CO}_{2} \text { eq }\right)\end{array}$ \\
\hline Polipropileno & $\mathrm{kg}$ & 1000 & 1.7858719 & 1785.87190000 \\
\hline Aceite lubricante & $\mathrm{kg}$ & 0.00015 & 1.0458843 & 0.00015688 \\
\hline Acero & $\mathrm{kg}$ & 0.0018 & 0.4307997 & 0.00077544 \\
\hline Energía & $\mathrm{kWh}$ & 950 & 0.3072000 & 291.84000000 \\
\hline Producción LDPE & $\mathrm{kg}$ & 0.0195 & 1.8637658 & 0.03634343 \\
\hline Residuos LDPE & $\mathrm{t}$ & 0.0000195 & 21.3842000 & 0.00041699 \\
\hline \multicolumn{5}{r}{ TOTAL (por t de tubo protector) } \\
\hline
\end{tabular}

Este dilema sobre los distintos impactos ambientales también está presente en la gestión de residuos. Los factores de emisión determinan que el tratamiento en vertedero tiene menor impacto respecto al cambio climático que cualquier tipo de reciclaje o incineración (un 60\% menos de emisiones). Sin embargo, los vertederos pueden producir contaminación de suelos y aguas subterráneas. Por tanto, es recomendable realizar un análisis de ciclo de vida completo para tomar ciertas decisiones, de esta manera sopesando todos los impactos ambientales.

Por último, los cálculos de las absorciones confirman la diferente capacidad de la masa forestal dependiendo de su composición. La masa productora, al estar compuesta de una especie de crecimiento rápido, puede absorber casi tres veces más que la repoblación multiusos en un año (véase Tabla 3). De todas formas, la HC del proceso repoblador se compensa en ambos casos en menos de 3 meses, y representan menos de un 1\% de las absorciones totales.

\section{Conclusiones}

Con este trabajo se ha cumplido el objetivo de facilitar la cuantificación de la $\mathrm{HC}$ de las repoblaciones forestales, proceso difícil por la escasez de inventarios del ámbito forestal. Aunque haga falta que se elaboren más inventarios de calidad, la herramienta elaborada puede ser utilizada por las empresas y organizaciones públicas que deseen llevar a cabo un proyecto de repoblación forestal para compensar sus emisiones, aumentando así la sensibilidad de sus informes de sostenibilidad.

Se concluye que la actividad de la maquinaria y la producción de los tubos protectores suponen las mayores aportaciones de emisiones de la $\mathrm{HC}$ de una repoblación, pero el impacto es tolerable dada la rapidez con la que se compensan dichas emisiones a través de las absorciones de las masas forestales creadas. 


\section{Agradecimientos y financiación}

Este trabajo ha sido parcialmente financiado por el Gobierno de la Comunidad de Madrid a través del Proyecto REMEDINAL-TE-CM (S2018-4338) y, parcialmente, por el Gobierno de España a través del Proyecto FORADMIT (AGL201677863-R).

Asimismo, se ha realizado dentro del marco de Campus Sostenible de la Universidad Politécnica de Madrid, financiado con la beca de sostenibilidad RES2+U.

\section{Bibliografía}

Arnold, J. and Alston, S., 2012. Life cycle assessment of the production and use of polypropylene tree shelters. Journal of Environmental Management, 94(1), pp.1-12. https://doi.org/10.1016/j.jenvman.2011.09.005

Falkowski, P., 2000. The Global Carbon Cycle: A Test of Our Knowledge of Earth as a System. Science, 290(5490), pp.291-296. https://doi.org/10.1126/science.290.5490.291

Grupo TRAGSA, 2020. Tomo II Tarifas 2020 para encomiendas a Tragsa no sujetas a impuestos en el territorio nacional sin Canarias: Grupo F Trabajos Forestales y Medioambientales.

TRAGSA Empresa de Transformación Agraria.
http://tarifas.tragsa.es/prestowebisapi.dll?FunctionGo\&id=1415365\&cod=TRAGSA20201.0879/F\&path=Tragsa2020W-Act-no-sujetas-Trgsa-Resto.cfg. Accedido 10 octubre 2020.

Lippke, B., Oneil, E., Harrison, R., Skog, K., Gustavsson, L. and Sathre, R., 2011. Life cycle impacts of forest management and wood utilization on carbon mitigation: knowns and unknowns. Carbon Management, 2(3), pp.303-333. https://doi.org/10.4155/cmt.11.24

Liu, X., Yang, T., Wang, Q., Huang, F. and Li, L., 2018. Dynamics of soil carbon and nitrogen stocks after afforestation in arid and semi-arid regions: A meta-analysis. Science of The Total Environment, 618, pp.1658-1664. https://doi.org/10.1016/j.scitotenv.2017.10.009

Serrada, R., Montero, G. and Reque, J., 2008. Compendio de selvicultura aplicada en Espan_a. [Madrid]: Instituto Nacional de Investigacio_n y Tecnologi_a Agraria y Alimentaria, Ministerio de Educacio_n y Ciencia, pp.117-154.

Taylor, S. and Marconi, S., 2020. Rethinking global carbon storage potential of trees. A comment on Bastin et al. (2019). Annals of Forest Science, 77(2). https://doi.org/10.1007/s13595-0200922-z

Wernet, G., Bauer, C., Steubing, B., Reinhard, J., Moreno-Ruiz, E., and Weidema, B., 2016. The ecoinvent database version 3 (part I): overview and methodology. The International Journal of Life Cycle Assessment, [en línea] 21(9), pp.1218-1230. https://doi.org/10.1007/s11367016-1087-8 